\title{
Partially-Covered Fractal Induced Turbulence On Fins Thermal Dissipation
}

\section{Soon Hong Chew}

Monash University Malaysia

\section{Su Min Hoi}

Tunku Abdul Rahman University College

Manh-Vu Tran

Monash University Malaysia

Ji Jinn Foo ( $\triangle$ Foo.Ji.Jinn@monash.edu )

Monash University Malaysia

\section{Research Article}

Keywords: Fractal geometry, Turbulence, Heat transfer, Plate-fin heat sink, Power spectra density, Vortex shedding

Posted Date: November 1st, 2021

DOl: https://doi.org/10.21203/rs.3.rs-965861/v1

License: (c) (1) This work is licensed under a Creative Commons Attribution 4.0 International License. Read Full License 


\title{
Partially-covered fractal induced turbulence on fins thermal dissipation
}

\author{
Soon Hong Chew ${ }^{1 *}$, Su Min $\mathrm{Hoi}^{2}$, Manh-Vu Tran ${ }^{1}$ and Ji Jinn Foo ${ }^{1 *}$ \\ ${ }^{1}$ School of Engineering, Monash University Malaysia, 47500, Bandar Sunway, Malaysia \\ ${ }^{2}$ Faculty of Engineering and Technology, Tunku Abdul Rahman University College, 53300 \\ Kuala Lumpur, Wilayah Persekutuan Kuala Lumpur, Malaysia \\ *Corresponding author: soon.chew@monash.edu, Foo.Ji.Jinn@monash.edu
}

\begin{abstract}
The impacts of partially-covered fractal grids induced turbulence on the forced convective heat transfer across plate-fin heat sink at Reynolds number $\operatorname{Re}_{D h}=22.0 \times 10^{3}$ were numerically and experimentally investigated. Results showed that partially covered grids rendered a higher thermal dissipation performance, with partially covered square fractal grid (PCSFG) registering an outstanding increase of $43 \%$ in Nusselt number relative to the no grid configuration. The analyzation via an in-house developed single particle tracking velocimetry (SPTV) system displayed the findings of unique "Turbulence Annulus" formation, which provided a small degree of predictivity in the periodic annulus oscillations. Further assessments on PCSFG revealed the preferred inter-fin flow dynamics of (i) high flow velocity, (ii) strong turbulence intensity, (iii) vigorous flow fluctuations, (iv) small turbulence length scale, and (v) heightened decelerated flow events. Furthermore, power spectra density unveiled the powerful vortex shedding effect, with PCSFG achieving fluctuation frequency $f=18.5 \mathrm{~Hz}$ close to an optimal magnitude. Such intricate flow structures pave the way for superior thermal transfer capabilities, benefiting the community in developing for higher efficiency heat transfer systems.
\end{abstract}

Keywords: Fractal geometry; Turbulence; Heat transfer; Plate-fin heat sink; Power spectra density; Vortex shedding

\section{Introduction}

Turbulences are described as flows that possess irregular, unpredictable and chaotic fluid motions. The formation of turbulence links closely to the behaviour of particles, whereby excessive kinetic energy in portions of fluid are able to overcome the viscosity effects that dampens flow fluctuations ${ }^{1}$. It is encountered in everyday phenomenon and has great mixing capabilities due to intrinsic diffusive characteristics of increased rate of mass, momentum and energy transport. Such mixing properties heightened the flow-thermal boundary layers reconstruction and reshuffling probability, thus enhancing forced convection. To date, 
multitudinous approaches had been conducted to unravel the heat transfer oriented flow patterns. The usage of 2D planar space-filling fractal grids have seen an increase in reputation for its effectiveness as a turbulator, owing to the feasibility of fine-tuning grid geometries in expressing for a preferred thermo-fluid interplay.

The aforementioned planar fractal grids consist self-similar geometries of various scales, with the pioneering study conducted by Hurst and Vassilicos ${ }^{2}$. Recently, the turbulators are seen in wide area of investigations, which includes but not limited to impinging jets ${ }^{3-5}$, flame speed augmentation ${ }^{6}$, and energy harvesting ${ }^{7,8}$. Square fractal grids (SFG) in particular have been extensively researched due to the distinctive nature of turbulence production and decay regions ${ }^{5,9}$. It was reported that the turbulence generated from multilength-scale fractal grids attained higher turbulence intensities and local flow Reynold number Re with reference to a typical regular grid of similar or higher blockage ratio $\sigma_{r}{ }^{10,11}$. Wakes shed by the grid bars of different length-scales meet at different downstream distances, and thus elongating the turbulence production region. Such flow structures enable higher heat transfer capabilities.

However, the hindrance from multitudinous wake interactions could possibly retard the airflow velocity. Hence, it raised the following questions: Is there any other fractal designs that could better enhance heat dissipation performance? Will it be possible to maintain the advantages of SFG induced turbulences, whilst improving heat sink thermal transfer, even at a targeted localized region? What will be the corresponding fluid flow structures induced through such newly designed grid? The outcome of these queries would propose a novel grid model that would provide additional insights in the preferred flow dynamics for thermal dissipation. For the current investigation, it was hypothesized that a vertical segmentation to SFG may amass the production of highly positive thermal dissipation flow structures that is advantageous for the forced convective heat transfer of a plate-fin heat sink, paving the way for the development of high efficiency heat transfer systems.

\section{Methods}

Current investigation offers the means of acquiring an in-depth realization of positive thermal dissipative flow characteristics. As such, numerical and experimental approaches will be utilized to deduce and explain the grid-induced plate-fin heat transfer performances, as well as the underlining inter-fin flow dynamics. Briefly, a transparent acrylic wind tunnel of dimension $160 \times 160 \times 2560 \mathrm{~mm}^{3}$ was connected to a flow-straightened bell mouth inlet, with an axial fan (Kruger, SG) paired along to ensure centreline inlet air velocity $U_{0}=2 \mathrm{~ms}^{-1}$, 
corresponding to Reynold number of $\operatorname{Re}_{D h}=22.0 \times 10^{3}$. The current use of turbulator includes (a) regular grid (RG), (b) square fractal grid (SFG), (c) partially-covered regular grid (PCRG) and (d) partially-covered square fractal grid (PCSFG) as shown in Fig..1(a-d), with no grid (NG) configuration acting as the control. The grids enjoy similar blockage ratio $\sigma_{r}=0.49$ and details of the grids' dimensions, i.e. thickness ratio $t_{r}$ and fractal iterations $N$ can be observed in Table 1. Each grid configuration together with a $1 \times 4$ rectangular plate-fin heat sink (aluminium 1100H14) of dimension $4 \times 20 \times 160 \mathrm{~mm}^{3}$ fabricated symmetrically to a comparable heated base plate were positioned within the wind tunnel test section as shown in Fig. 1(e), with the inter-fin spacing $\delta$ and grid-fin distance $l$ deduced at $5 \mathrm{~mm}$ and $10 \mathrm{~mm}$, respectively. A heater plate (GUNT, DE) was utilized to provide constant base heat flux of $q^{\prime \prime}=6 \times 10^{3} \mathrm{Wm}^{-2}$. As such, the instantaneous temperatures at locations illustrated in Fig. 1(f) were measured using seven $T$ type thermocouples and recorded through a data logger (GL800,.US) for a span of five minutes (steady-state). The average Nusselt number $\mathrm{Nu}$ of the plate-fin was then calculated with Eq. (3) to empirically evaluate the performance of forced convection, as shown below:

$$
\begin{gathered}
D_{h}=\frac{4 A_{w}}{P} \\
\Delta T=T_{m}-\frac{T_{\text {in }}+T_{\text {out }}}{2} \\
N u=\frac{q^{\prime \prime} D_{h}}{k_{\text {air }} \Delta T}
\end{gathered}
$$

where $D_{h}$ denotes the hydraulic diameter, $A_{w}$ the wind tunnel cross-sectional area, $P$ the crosssectional perimeter, $T_{m}$ the mean temperature of plate-fin heat sink, $T_{\text {in }}$ the inflow temperature, $T_{\text {out }}$ the outflow temperature, and $k_{\text {air }}$ the air thermal conductivity.

\begin{tabular}{|c|c|c|c|c|c|c|c|}
\hline \multirow{2}{*}{ Grid type } & \multirow{2}{*}{$t_{r}$} & \multicolumn{2}{|c|}{$N=1$} & \multicolumn{2}{|c|}{$N=2$} & \multicolumn{2}{|c|}{$N=3$} \\
\hline & & $L_{0}(\mathrm{~mm})$ & $t_{0}(\mathrm{~mm})$ & $L_{1}(\mathrm{~mm})$ & $t_{1}(\mathrm{~mm})$ & $L_{2}(\mathbf{m m})$ & $t_{2}(\mathrm{~mm})$ \\
\hline RG & 1.00 & 160.00 & 5.76 & - & - & - & - \\
\hline PCRG & 1.00 & 160.00 & 6.50 & - & - & - & - \\
\hline SFG & 9.77 & 101.43 & 24.45 & 50.71 & 5.00 & 22.86 & 2.50 \\
\hline PCSFG & 9.77 & 101.43 & 24.45 & 53.71 & 8.00 & 22.86 & 2.50 \\
\hline
\end{tabular}

82 Table 1

83 Parameters of the grids in Fig. 1(a-d). 
The setup was also modelled as a computational domain in numerical investigation using the computational fluid dynamics (CFD) commercial software package ANSYS-Fluent (ver..16.0, USA). Reynold Stress Model (RSM) was employed under the first order upwind spatial discretization to offer the prediction of grid-induced turbulence. The convergence criteria of residual $10^{-3}$ were prescribed for all the governing computational calculations, with the exception of energy equation to be $10^{-6}$. Details of the governing equations can be referenced in Teh et al. ${ }^{12}$. Besides, mesh independency test using finer tetrahedron elements around the grid-fin conformation was also conducted, with maximum percentage difference of $0.48 \%$ recorded for $\mathrm{Nu}$ as the number of elements increased from $0.8 \times 10^{6}$ to $3.0 \times 10^{6}$. In all cases, reliable numerical outcomes were secured. Furthermore, two main parameters, namely $(\delta, l)$, were explored for the design of experiment (DoE) to systematically observe the individual influence and correlation with respect to grid-fin's $\mathrm{Nu}(\delta, l)$. A total of 55 sampling points were customary input to the design space, and Kriging regression was applied to predict the response surface mapping with respect to CFD processed DoE data points.

Subsequently, a re-scaled transparent acrylic plate-fin setup (replacing aluminium heat sink) was employed together with an in-house developed single particle tracking velocimetry system (SPTV) to empirically capture grid-induced flow fluctuations. SPTV introduced the means of investigating localized flow structures in a non-intrusive and inexpensive approach. It captures the concatenating spatial position of a tracer particle that is lightly attached to a polyester yarn fluctuating at an inter-fin 'local' region with a pair of synchronized high-speed cameras [see Fig. 1(f)]. The particle was imaged by two coupled charged devices (CCD) cameras (FLIR Integrated Imaging Solution Inc., CA) on top and side sections of wind tunnel at $80 \mathrm{fps}$, resulting in the compilation of $4.82 \times 10^{3}$ images per camera. Image processing and correction methods were then performed using internally established MATLAB (R2016b, US) algorithm to acquire highly contrast particle images that were exempted from cameras' distortion, refractive and perspective misplays. Such features allowed for the accurate detection of particle's centroid in consecutive images through built in computational algorithm. Through comparison on the locality of tracer particle in successive images, the spatial position can be reconstructed in a Cartesian coordinate system, where the particle's trajectory, velocity fluctuations along with the underlining flow dynamics can be computed accurately. Details of the calibration and correction processes are shown in the following sections. 
Two synchronized high-speed cameras were calibrated to ensure the precise alignment

117 of optical axes at orthogonality with one another by incorporating a calibration platform

118 [see.Fig..2(a)]. The calibrator consisted of 77 white circles uniformly arranged at a $7 \times 11$ array

119 in both $X-Y$ and $X-Z$ planes, where frontal images of the circles were captured and analysed

120 using computational algorithm to attest for the maximum deviation of $0.10 \mathrm{~mm}$ between optical

121 axis and platform centre, thus assuring parallelism of cameras' optical plane with the platform.

122 The intersection between the optical axes denotes the origin of the coordinate system, with

$123(x, y, y)$ representing the streamwise, spanwise and transverse directions, respectively.

124 Thereupon, the particle's fluctuating positions were captured. The individual time-series

125 images were further processed by an un-distortion algorithm in MATLAB single-camera

126 calibration toolbox to minimize distortion effects. Briefly, images of checkerboard with square

127 arrays of $5 \times 8$ were first recorded at 20 different orientations using the two cameras and

128 registered subsequently in the toolbox. Consequently, the calibration parameters, i.e. focal

129 length, optical centre and lens distortion coefficient were pinpointed, whereby a custom

130 algorithm was realized to undistort all images, securing the SPTV particle's spatial accuracy.

131 The aforementioned images were further processed to increase the contrast of the tracer

132 particle through background subtraction and bitonal conversion techniques. Individual pixels

133 of the images embodied a pixel value that varies between 0 (black) to 255 (white), with

134 numerals in between representing monochrome colour tones. Each pixelized value was

135 subtracted with the background (image with absence of tracer particle) to minimize background

136 noises. Subsequently, an intensity threshold was introduced to binarize the images, i.e. setting

137 pixel value above threshold to 1 (white) and vice versa, so that the contrast of tracer particle

138 was emphasized, thus attaining a high signal-to-noise image for particle identification. The

139 centroid of the particle was then determined by employing circular Hough transform, which is

140 accessible through the MATLAB built-in function.

The centroid of tracer particle determined at current stage is subjected to refractive and projection errors. As the correction processes for both cameras are similar, only the side camera

143 will be referred in the discussions hereafter. Note that coordinate notations $(x, z)$ are utilized

144 for side camera and $(x, y)$ for top camera. Concisely, refractive errors occur due to the bending 145 of light as it passes between mediums of different optical densities. The detected particle 146 positions will be observed to offset from the actual position, as illustrated in Fig. 2(b). As such, 
a corrective module that utilizes Snell's law had been incorporated into the MATLAB algorithm to tackle the inaccuracies. The equations utilized in the module are observed as below:

$$
\begin{gathered}
\bar{R}=\sqrt{\left(x_{d}-x_{c}\right)^{2}+\left(z_{d}-z_{c}\right)^{2}} \\
\theta_{1}=\tan ^{-1}\left(\frac{\bar{R}}{y_{D}+y_{w}+y_{l}}\right) \\
\theta_{2}=\sin ^{-1}\left(\frac{n_{1} \sin \theta_{1}}{n_{2}}\right) \\
R=y_{D} \tan \theta_{1}+y_{w} \tan \theta_{2}+y_{l} \tan \theta_{1}
\end{gathered}
$$

149 where $\left(x_{c}, z_{c}\right)$ represents the coordinates of optical centre, $\left(x_{d}, z_{d}\right)$ the camera detected particle

150 centroid, $\bar{R}$ the observed displacement from optical centre, $y_{D}$ the detected horizontal particle151 to-wall distance, $y_{w}$ the acrylic wall thickness, $y_{l}$ the horizontal lens-to-wall distance, $\left(\theta_{l}, \theta_{2}\right)$ 152 the Snell's law angles, $\left(n_{1}, n_{2}\right)$ the refractive indices of (air, acrylic), and $R$ the corrected 153 displacement from optical centre. Briefly, the $\left(x_{d}, z_{d}\right)$ were coded as the input to initiate a series 154 of computational equations that allowed for the $R$ to be acquired. Intrinsically, coordinates corrected for refractive error $\left(x_{r}, z_{r}\right)$ can be determined through rationalizing for similar triangles, and are computed as follow:

$$
\begin{aligned}
& x_{r}=x_{c}+\left(x_{d}-x_{c}\right) \cdot \frac{R}{\bar{R}} \\
& z_{r}=z_{c}+\left(z_{d}-z_{c}\right) \cdot \frac{R}{\bar{R}}
\end{aligned}
$$

On the other hand, the perspective distortion caused different magnification of particle image in relative to the distance from lens [see Fig. 2(c)]. Notably, the pixel-to-distance ratio varied at different planar locations, where particle situated closer to the lens would appear to be enlarged, and a small shift in particle coordinate would emerge as a large motion as detected by camera. Such repercussions were minimized by uncovering the perspective projection equations. Generally, all the time-series images of tracer particle were perceived to be projected onto a focusing frame coincident with the calibration platform. Since the dimensions of the calibration platform were discerned, the metric distance of the particle from optical centre can be calculated through pixel-to-length conversion. By locating the depth of particle in relative to the camera lens, the correct metric scale can be determined, hence an accurate spatial coordinate reconstruction can be computed as below: 


$$
\begin{aligned}
& x_{a}=\frac{y_{D}+y_{w}+y_{l}}{y_{f}+y_{w}+y_{l}}\left(x_{r}-x_{c}\right)+x_{c} \\
& z_{a}=\frac{y_{D}+y_{w}+y_{l}}{y_{f}+y_{w}+y_{l}}\left(z_{r}-z_{c}\right)+x_{c}
\end{aligned}
$$

168

169

170

171

172

173

174

where $y_{f}$ represents the frame-to-wall distance, and $\left(x_{a}, z_{a}\right)$ the calculated coordinate of the tracer particle.

For the algorithm to be fully functional, the information for $y_{D}$ is imperative. However, said variable obtained directly from the top camera would still be subjected to perspective errors. Therefore, geometrical formula is used to pinpoint the $y$-coordinate $\left(y_{a}\right)$ of the particle such that the inaccuracy of $y_{D}$ can be minimized. By observing on Fig. 2(d, e), the $\left(y_{a}, z_{a}\right)$ coordinates can be related by similar triangle equations:

$$
\begin{aligned}
& y_{a+1}=\frac{y_{r}-y_{c}}{z_{l}+z_{w}+z_{f}}\left(z_{l}+z_{w}+0.5 z_{f}-z_{a}\right)+y_{c} \\
& z_{a+1}=\frac{z_{r}-z_{c}}{y_{l}+y_{w}+y_{f}}\left(y_{l}+y_{w}+0.5 y_{f}-y_{a}\right)+z_{c}
\end{aligned}
$$

175 An iterative method had been utilized until both coordinates converge with a criteria of $176 \varepsilon=1.0 \times 10^{-2} \mathrm{~mm}$. Combining information from both cameras, an accurate particle coordinate $177\left(x_{a}, y_{a}, z_{a}\right)$ can finally be realized. As such, the instantaneous velocity fluctuation in the three 178 different directional components can be computed. It was calculated by the change of 179 displacement over a small time interval between frames, through the equations listed below:

$$
u^{\prime}=\lim _{t_{2} \rightarrow t_{1}} \frac{x_{2}-x_{1}}{t_{2}-t_{1}} \quad v^{\prime}=\lim _{t_{2} \rightarrow t_{1}} \frac{y_{2}-y_{1}}{t_{2}-t_{1}} \quad w^{\prime}=\lim _{t_{2} \rightarrow t_{1}} \frac{z_{2}-z_{1}}{t_{2}-t_{1}}
$$

180 where $u^{\prime}, v^{\prime}$ and $w^{\prime}$ denote the velocity fluctuations in the $(x, y, z)$ directions, $t$ the image 181 timestamp and subscript $(1,2)$ the parameters on the current and subsequent frame, respectively. 182 The employment of SPTV allows for the detection of turbulence characteristics, and are further 183 extracted for analysis purposes. 


\section{Results and Discussions}

\section{RSM and SPTV validation processes}

In order to confirm the numerical accuracy of current RSM in revealing the fundamentals of insert-induced turbulence upon fins forced convection, careful validation using Hoi et al.'s ${ }^{13,14}$ experimentally recorded measurements are conveyed. Fig. 3(a) demonstrates the comparison between the experimental study of NG and SFG induced Nu with present numerical simulation. Evidently, small percentage differences of $0.34 \%$ and $0.19 \%$ are recorded for NG and SFG, respectively. Likewise, fractal grid generated flow dynamic, namely, the centreline streamwise flow velocity $U_{c}$ is as well validated and compared with experimental data. It is seen in Fig. 3(b) that the normalized flow velocity at the lee of grid decreases $\times 0.28$ nonlinearly, i.e., from about $U_{c} / U_{0}=1.9$ to 1.4 , with a maximum discrepancy of $6.15 \%$ recorded between the numerical and experimental results. Such subtle documented differences in Fig..3(a, b) indicate that the RSM simulated forced convection and fluid flow fits practically well with the experimental study. Hence, it is justified that the current use of numerical scheme is capable of predicting grid-induced flow dynamic in aiding the thermal dissipation of platefin heat sink with reasonable accuracy.

Similarly, validation on the accuracy of the current SPTV coordinate detection and reconstruction system is also conducted. The SPTV tracer particle had been manually complemented into the wind tunnel test section at known spatial positions. Fig. 3(c) demonstrates the careful arrangement of tracer particle along the $(x, y, z)$ axes, with each placement bearing a step size of $1 \mathrm{~cm}$, expanse from the origin. Subsequently, particle images are recorded at each coordinate and analysed using MATLAB algorithm, whereby the error margins can be computed [see Fig. 3(d)]. Clearly, the maximum percentage errors attained for each directional component are discerned to be $1.49 \%, 1.69 \%$, and $1.62 \%$, respectively, which corresponds to the highest recorded inaccuracy of $5.0 \times 10^{-2} \mathrm{~cm}$. Such minute misplays imply a high accuracy and reliability in spatial reconstruction, thus validating the accuracy of current SPTV in coordinate detection.

\section{Effects of $\delta$ and $l$ on fins forced convection}

From the numerical study, the correlations between $\delta$ and $l$ upon plate-fin heat sink forced convection at $\operatorname{Re}_{D h}=22.0 \times 10^{3}$ are acquired. As seen in Fig..4(a), the RG induced Nu expresses its unique forced convection as a function of $\mathrm{Nu}(\delta, l)$ with a wider high $\mathrm{Nu}$ coverage amongst all grid configurations that spans between $4.0 \mathrm{~mm} \leq \delta \leq 35.5 \mathrm{~mm}$ and $10 \mathrm{~mm} \leq l \leq 75.5 \mathrm{~mm}$. 
$216 \mathrm{RG}$ achieved a rather consistent $\mathrm{Nu}$ distribution, owing to the uniform $t_{0}$ assignment that 217 generates wakes of highly homogenous and isotropic turbulence accompanied with lower flow 218 dynamic disturbance. Such weaker wake interplays lessen the disruption to flow boundary layer 219 along each fin, hence justifying the all-around lower capability in promoting thermal 220 dissipation. On another note, SFG is observed to attain an extensive high Nu coverage in comparison with RG, but smaller in the expanse of partially-covered grids [see Fig. 4(b)]. Such enhancement in $\mathrm{Nu}$ contrasting to $\mathrm{RG}$ is likely to be contributed via the multi-length scale interactions of wakes that originated from SFG's variety fractal bar thicknesses. Clearly, increasing the parameters $(\delta, l)$ is observed to have deteriorating effects in $\mathrm{Nu}$, indicating that the thermal dissipation performance is highly sensitive to the tuning of $\delta$ and $l$. Moreover, a weak $\mathrm{Nu}$ regime is determined at the span of $15 \mathrm{~mm} \leq \delta \leq 25 \mathrm{~mm}$ and $10 \mathrm{~mm} \leq l \leq 14 \mathrm{~mm}$. The phenomenon manifests similar observation in preceding findings $\mathrm{s}^{15}$, where the deterioration is instituted from weak turbulence intensity and low flow velocity between fins.

Interestingly, PCRG and PCSFG are able to realize an effective domain of high thermal 230 dissipation, with higher $N u(\delta, l)$ discerned around $4 \mathrm{~mm} \leq \delta \leq 10 \mathrm{~mm}$ for the former and $2314 \mathrm{~mm} \leq \delta \leq 12 \mathrm{~mm}$ the latter [see Fig. 4(c, d)]. The integration of larger $t_{0}$-induced wakes 232 (see.Table 1) accompanied with fluid flow acceleration through the vertically aligned separation generates beneficial hydrodynamic interactions which gives rise to intense forced convection. Notably, the $\mathrm{Nu}$ is less sensitive towards $l$. The partially-covered grids induced flow perturbation brings about the highest local acceleration immediately leeward from grid. The lengthening of $l$ increases the jet mixing in terms of spanwise and streamwise flow dynamic dissipation and diffusion which eventually retard slightly the fins' thermal transfer.

238 Yet, $\mathrm{Nu}$ decreases with increasing $\delta$. The penetration of flow fluctuations into fins' flow 239 boundary layer may have been gradually weaken at larger $\delta$, which supports unwanted fluid 240 bypass. Surprisingly, PCSFG is able to mitigate the shortcoming of SFG, where the lowest $\mathrm{Nu}$ generated is observed at a region of much larger $\delta$ and $l$ (blue). The high flow velocity penetrated through the perforations and central void reinforces the favourable multitudinous wake interactions initiated from fractal bars of different length scale, effectively empowering thermal dissipation performance. In general, the $2 \mathrm{D} \mathrm{Nu}$ contours suggest that the implementation of partially-covered grids are capable of providing higher thermal dissipation even at a wider $\delta$. Hence, the optimum $\delta=5 \mathrm{~mm}$ and $l=10 \mathrm{~mm}$ is empirically evaluated in the next section for a deeper insight on the various grid-induced flow dynamics. 
Undoubtedly, the experimentally proven highest $N u=4341.7$ is achieved using PCSFG as shown in Fig. 4(e), with a remarkable percentage enhancement of $42.9 \%$, as compared with the control NG. The augmentation in $\mathrm{Nu}$ is followed by PCRG, SFG, and RG ranked in descending order, with the percentage increase recorded as $29.2 \%, 21.0 \%$ and $12.8 \%$, respectively. The attainment of $\mathrm{Nu}$ induced from different turbulators are comparable to the numerical results performed through RSM, thus uncovering the value of simulated studies in predicting for the trend of forced convective heat transfer on plate-fin heat sink through various 2D planar grids. Clearly, the current uses of partially-covered grids are superior to their classic grid counterparts in terms of heat transfer, suggesting the implementation of a vertical midplane separation incites favourable flow dynamics that support forced convection.

On another note, the localized Nusselt number $\mathrm{Nu}_{L}$ at locations mentioned in Fig. 1(f) are illustrated in Fig. 4(f, g) to identify the locations with the utmost heat transfer. Interestingly, the $\mathrm{Nu}_{L}$ displayed at the base of plate-fin for all grid configurations showed symmetricity, whereby the $\mathrm{Nu}_{L}$ surges at the base of mid fin (position 2). The highest achieved $\mathrm{Nu}_{L}(2)$ is recorded for SFG owing to the presence of thick $t_{0}$ assignment, which generates wakes of sizeable length-scale near the base region for vigorous thermo-fluid interplay. Yet, the effects slowly diminished as we shift the position to centreline, whereby PCSFG is now observed in Fig. $4(\mathrm{~g})$ to dominate the forced convection through realization of potent $\mathrm{Nu}_{L}$ at inter-fin regions (positions 4, 5 and 6). In spite of the high attainment of $\mathrm{Nu}_{L}$, a steep decline is observed near the outer-fin surface (position 7) for SFG, PCRG and PCSFG. Current phenomenon might indicate the diverging of airflow afar from fins' exterior, owing to the growth of flow boundary layer at leading edge of outer-fins. However, a dissimilar phenomenon is observed for RG, where $N u_{L}(7)$ is noticed to be comparable with $N u_{L}(5)$ but demonstrate a deterioration in $N u_{L}(6)$.

272 The evenly separated RG perforations with uniform $t_{0}$ generated an alternating region of high and low airflow velocity $U$ lee of grid, producing an imprint of $t_{0}$ in dissociating for retardation of flow. Such imprint is projected streamwise towards the fin, with position 6 likely to be coincident with it. The low flow kinetic energy reduces the likelihood of flow reshuffling, thus sabotaging the plate-fin local thermal dissipation. On another note, positions $(5,7)$ are integrated at the immediate region behind grid perforations, which surges the $N u_{L}$ due to the heightened flow acceleration. Nevertheless, the forced convection is noticeably greater at the mid of fins, i.e. positions $(2,5)$ for all grid conformations. Therefore, the SPTV tracer particle 
is implemented at centreline $x / D_{h}=0.125$ to investigate the flow dynamics that dominates thermal transfer processes.

Fig. 5(a) illustrates the $U$ normalized with $U_{0}$, i.e. $U / U_{0}$ induced through the different 2D planar grids. The $U$ is gauged by means of a hotwired anemometer (Testo 405i, DE) at centreline $x / D_{h}=0.125$ in the absence of plate-fin array. Notably, the $U / U_{0}$ generated through various grids exhibit similar trend as the $\mathrm{Nu}$, with $U(\mathrm{PCSFG})>U(\mathrm{PCRG})$, and $U(\mathrm{SFG})>U(\mathrm{RG})$. It is renowned that the implementation of turbulator allows the acceleration of air flow due to the sudden contraction of flow passage. The introduction of a vertically aligned separation in the partially-covered grids promotes mid-plane jet formations, owing to the principle of mass conservation. Such separation effectively reduces the undesirable fluid bypass around the platefins, and forcefully regulates the working fluid to penetrate inter-fin regions. In addition, the accelerated airflow in between the fin array enforces greater wall shear stress along fin surfaces, which limits the growth of viscous sub-layer, thus effectively enhancing forced convection.

Even though the mid-plane separation is employed on both PCRG and PCSFG, a greater heat dissipation is still documented for the latter. The enhancement in Nu for PCSFG contrasting with PCRG may be due to the multi-length scale wake interactions via the multiplicity of fractal bar thickness. As reported earlier ${ }^{9,16}$, the interplay between accelerated airflow and wake structures through the variety of fractal bar thickness promotes generation of multi-length scale turbulent eddies. Small-scale eddies induced through thinner fractal bars are described to effectively facilitate plate-fin heat dissipation through flow dynamic energy cascading. The formation of anisotropic and inhomogeneous eddy structures encompassing a collection of sizes and frequencies increase the likelihood to the disruption of flow boundary layers along the fin. On the contrary, PCRG engenders weaker flow agitation capabilities due to the isotropic and homogenous turbulence formation by uniform $t_{0}$ assignment. With the current employment of PCSFG, effects of conventional fractal grids with the added benefit of pronounced airflow acceleration at a localized region are amalgamated. The critical fragments of turbulent eddies may be filtered along the inter-fin regions, coupling with the high $U / U_{0}$ triggers the active reshuffling of flow boundary layers, thus expounding the event where $N u(\mathrm{PCSFG})>N u(\mathrm{PCRG})$. 

the formation of turbulence eddies. In order to quantify the regional turbulence intensity $I$, the

312 root mean square of velocity fluctuations detected from SPTV is rationalized with $U_{0}$ :

$$
I=\frac{\sqrt{\frac{1}{3}\left(\left\langle u^{\prime 2}\right\rangle+\left\langle v^{\prime 2}\right\rangle+\left\langle w^{\prime 2}\right\rangle\right)}}{U_{0}}
$$

313 where $\left\langle u^{\prime 2}\right\rangle,\left\langle v^{\prime 2}\right\rangle$ and $\left\langle w^{\prime 2}\right\rangle$ represent the ensemble average of squared velocity fluctuations 314 in $(x, y, z)$ directions, respectively. As illustrated in Fig. 5(b), the paradigm demonstrated by $I$ 315 for the various grids followed closely to that of $\mathrm{Nu}$, implying a positive correlation in between 316 the two, i.e. higher $I$ give rise to greater $\mathrm{Nu}$. Evidently, the $I$ induced from partially-covered grids are comparably more intense than that of the fully-covered grids, thereafter the control

318 NG. The raised level of $I$ documented for PCSFG and PCRG may be due to the restructuring of wake dimensions through implementation of a vertically-aligned separation, providing the energy necessary for vigorous turbulent eddies to infiltrate inter-fin regions.

In order to conceptualize the effects of $I$ induced from various grids, the trajectories of the tracer particles are demonstrated in Fig. 6(a,b), with the former representing the $Y-Z$ plane (cross-sectional view) and latter representing the $X-Z$ plane (side view). Clearly, smaller fluctuations are observed for NG and RG configurations, whereby the frontal coverage areas $A$ with respect to $Y-Z$ plane are recorded at a minimum of $1.2 \mathrm{~mm}^{2}$ and $1.4 \mathrm{~mm}^{2}$, respectively. Through the amalgamation of fractal bars, SFG is observed to attain a greater $A=2.9 \mathrm{~mm}^{2}$ that fairly resembles the shape of a crescent. Interestingly, both partially-covered grids develops an elliptical structure from the particle's trajectory, which would be fitting to addressed it as a 'Turbulent Annulus'. The formation of the annulus may signify the generation of new and unique flow structures, as it displays small degree of predictability in the particle's periodic annulus oscillations, despite the chaotic nature of turbulence. Nevertheless, higher fluctuation amplitude is observed from PCRG and PCSFG generated particle trajectory, especially in the $y$ - and $z$-directions, which are vital in disturbing the flow and thermal boundary layers. Remarkably, the $A$ of PCRG is greater than PCSFG despite the lower $I$ attainment. Distinct flow dynamics induced from PCSFG consolidated the particle's trajectory to a rather dense and intense flow fluctuation around the sizable annulus. Unlike for PCRG, which has a smaller dimension along with diffusive characteristic at the annulus interior. Such diffusive effect disperses flow energy to undesirable areas, thus retarding heat transfer processes. 

distribution on the $v^{\prime 2}$ and $w^{\prime 2}$ for the variety of turbulators are depicted in Fig..5(d, e). As shown in Fig. 5(d), NG and RG have a small range of spanwise fluctuations, whereby $95 \%$ of $v^{, 2}$ less than $3.8 \mathrm{~m}^{2} \mathrm{~s}^{-2}$ and $10.7 \mathrm{~m}^{2} \mathrm{~s}^{-2}$ are recorded, respectively. It showed a vast difference with SFG as $95 \%$ of $v^{\prime 2}$ is below $233.8 \mathrm{~m}^{2} \mathrm{~s}^{-2}$, implying a great elevation in fluctuation amplitude. However, the strength is still inferior to the partially-covered grids, with PCSFG recorded with a higher probability of stronger fluctuations approximately between $80.0 \mathrm{~m}^{2} \mathrm{~s}^{-2}<v^{, 2}<440.0 \mathrm{~m}^{2} \mathrm{~s}^{-2}$ as compared to PCRG. Likewise in Fig..5(e), the overall $w^{\prime 2}$ trend depicted minor differences with $v^{\prime 2}$, where a clear distinction of wider $w^{\prime 2}$ variations are still observed for PCSFG, along with PCRG, SFG, RG and NG ranked in descending order. The event is apparent at the top five percentile, as PCSFG realized the highest variation of $w^{, 2}>381.1 \mathrm{~m}^{2} \mathrm{~s}^{-2}$. The pronounced $v^{\prime}$ and $w^{\prime}$ give rise to greater development of $A$, suggesting a larger area of flow boundary is being agitated at the localized inter-fin region, thus poses a unique advantage in supporting potent thermal dissipation.

In the case of turbulent airflow, the effects of turbulence length scale $L$ on the forced convective heat transfer should be considered as well, as it unveils critical thermo-fluid interplay upon heat exchanger thermal transfer ${ }^{9,16} . L$ provides a representation of spatial dimension for turbulent vortices. Such $L$ are computed by integrating the normalized autocorrelation function of velocity fluctuation with respect to the time-lapse, using Eq. (17):

$$
\begin{aligned}
R_{f^{\prime}}(t) & =\frac{<f^{\prime}(t) f^{\prime}(t+\tau)>}{<f^{\prime}(t)^{2}>} \\
L & =U_{0} \int_{0}^{T} R_{f^{\prime}}(t) d \tau
\end{aligned}
$$

where $R_{f}$ 'represents the normalized autocorrelation function of velocity fluctuation, $f^{\prime}$ the directional components of velocity fluctuations $\left(u^{\prime}, v^{\prime}, w^{\prime}\right), \tau$ the time-lapse, $T$ the first zerocrossing of $R_{f}$, and < . > the ensemble average. The $L$ generated from the different 2D-planar grids are rationalized with $\delta$, i.e. $L / \delta$ and is shown in Fig. 5(c).

Unsurprisingly, the NG induced $L / \delta$ in $(x, y, z)$ directions are realized to be the highest. The straightened airflow is only subjected to wall shear stresses from wind tunnel surfaces, which lacks flow obstructions prior to plate-fin filtration, hence higher $L / \delta$. Conversely, the $L / \delta$ is substantially reduced with the use of space-filling grids: a direct consequence of grid bars in filling the planar spaces. Interestingly, RG and SFG developed a comparable $L(z) / \delta$, but are 
contrasting in reference to streamwise and spanwise $L$. The event suggests that a sizeable $L(y) / \delta$ by $\mathrm{SFG}$ is preferred for forced convection since $\mathrm{Nu}(\mathrm{SFG})>\mathrm{Nu}(\mathrm{RG})$, and could be enforced through the realization of smaller $L(x) / \delta$. By considering vortices rotating in the $X$ - $Z$ plane, the dwindling $L(x) / \delta$ may increase the vortices' angular velocity around $y$-direction $\omega_{y}$, but in turns extending the vortex line laterally due to the conservation of angular momentum, i.e. vortex stretching. The elongation of vortex structures thus effectively interacts and disrupts the fins' boundary layer. The aforesaid implication concurred closely with RG, yet poses an opposite effect of streamwise elongation, which lessened boundary layers' agitation probabilities.

Nevertheless, an overall small $L / \delta$ is still favourable for forced convective heat transfer, since partially-covered grids are noticed to induce a relatively small and uniform $L / \delta$. The findings are supported with reference to previous literatures ${ }^{15,17-19}$, whereby higher heat transfer is usually achieved at lower $L$. The contraction of flow due to the two partially-covered grid segments positioned along each side of wind tunnel could have developed a profound amount of similar yet counter-rotating pair of vortices. The vortex pair that oppose in motion maximize the straining of airflow, which induces highly intense eddies with smaller diameters. Such development of eddies could possibly be sorted along the fin surfaces, and promotes heat transfer through flow dynamic energy cascading.

In general, the coupling effect of $\left(U_{\text {high }}, I_{h i g h}, L_{l o w}\right)$ as demonstrated from PCSFG induces flow structures that are favourable in enhancing heat transfer. The results expressed in the current section showed that SPTV is viable to provide informative data to describe for the preferred thermal dissipation flow dynamics. However, one might wonder if the investigation on a targeted localized region using SPTV particle at centreline of inter-fin is sufficient to conclude for the whole plate-fin array. Hence, numerical predictions are utilized in the following to describe for the overall flow dynamics at inter-fin in terms of isosurfaces and 2D contours to append for the limitations of current experimental findings.

\section{Simulated flow properties at inter-fin region}

The isosurfaces of (i) $U / U_{0}=2.38$, (ii) $I=0.33$ and (iii) $2 \mathrm{D}$ contour of $L$ at $x / D_{h}=0.125$ are demonstrated in Fig. 7 to predict the underlying flow dynamics at a more pronounced interfin region. By focusing on RG in Fig. 7(a), it is noticed that the high $U / U_{0}$ is unlikely to penetrate through the plate-fin array. The evenly distributed perforations of RG generated low flow acceleration that is rapidly dissipated and dispersed with the surrounding tardy fluid momentum leeward of grid, disabling high $U / U_{0}$ to be regulated into the inter-fin separations. 
Besides, the isosurface distribution of strong $I$ in Fig. 7(b) is perceived to be partitioned into segments, with bigger voids arising between the second and third fins, owing to the $t_{0}$ imprints from windward grid geometry.

Moving on to SFG, it is noticed that the $3 \mathrm{D}$ isosurface of $U / U_{0}$ in Fig. 7(d) is able to penetrate well through the inter-fin separations. The incorporation of airflow that forced through SFG of thicker $t_{0}$ in conjunction with the unevenly distributed fractal dimension significantly stimulates the jet flows, producing working fluid of high flow velocity to predominantly infiltrate through the fin-array. Such flow structure increases the likelihood of flow perturbations, which contributed to the isosurface distribution of strong $I$ as shown in Fig..7(e). Interestingly, the isosurface distributions for both $U / U_{0}$ and $I$ are perceived to inhibit high isosurface porosity, with voids scattered towards the upper and bottom portions of each inter-fin. The flow recirculated in the lee from fractal bars incurs considerable flow retardation, yielding an imprint of $t_{0}$ dissociated isosurface. The amalgamation of such transition between the potent and frail interfaces may lessen the possible reshuffling of flow and thermal boundary layers along fins, which sabotage the local thermal dissipation.

Conversely, highly uniform $U / U_{0}$ isosurface scattering for the PCRG and PCSFG can be distinctly seen in Fig. 7(g, j), respectively. Apprehensively, jets are not only created and accelerated through the insert perforations via the sudden introduction of an incompressible grid, most importantly, the flow dynamics are as well simultaneously amassed around the midplane segmentation, which allows the air to speed up and penetrate deeper and more uniformly, hence increasing the scalar and flow momentum transportations. Moreover, denser isosurface distribution density can be clearly perceived with PCSFG as to PCRG. Notably, minor discontinuities are recorded for the former in comparison SFG, suggesting the effect of multitudinous wake interactions in lowering down the flow velocity has been significantly mitigated. The strong I distributions of PCRG and PCSFG are of greater extent with seemingly the least variation in terms of respective uniformity [see Fig. 7(h, k)]. Through effective coupling between the grid-induced turbulence and downstream plate-fin array, it initiates the second stage of flow eddies filtration, which leads to a predominant, unique fluid flow fluctuation along with potent flow dynamics to penetrate into fin-array. As a result, PCRG and PCSFG empower vigorous fin-wake interplay which effectively heighten forced convection. 


$$
L=\frac{C_{\mu}^{3 / 4} \kappa^{3 / 2}}{\varepsilon}
$$

431

432

433

434

435

436

437

438

439

440

441

442

443

444

445

446

447

448

449

450

451

452

453

where $\kappa$ denotes the turbulence kinetic energy, $\varepsilon$ the turbulence eddy dissipation rate, and $C_{\mu}$ a model constant of $C_{\mu}=0.09$. From Fig. $7(\mathrm{c}, \mathrm{f}), \mathrm{RG}$ configuration is clearly seen to incite the largest inter-fin $L$, whereas domain of non-uniformity can be observed with SFG. Admittedly, the region of high $L$ of both grids are associated with the corresponding $t_{0}$ of the windward grid geometry. The sizable span of $t_{0}$ gives rise to wakes of greater length scale, which generates substantial shear levels and subsequently bigger eddies via wake-flow hydrodynamic interplays. Conversely, SFG's smaller scale fractal bars promote the production of smaller eddies, that may be potently filtered and disseminated to the $t_{0}$ imprint complement regions. Interestingly, the small-scale eddies are determined in Fig. 7(i, l) to propagate and uniformly distributed for PCRG and PCSFG, similar to preceding findings in Fig. 5(c). The absence of first iterative fractal bar in the mid-plane segmentation eradicates the accumulation of high length scale wakes, thus preventing the formation of large turbulent eddies directly windward of fins. Most importantly, miniscule yet vital fragments of turbulent eddies are found to approach and attached actively along fin surfaces, which may effectively facilitate plate-fin heat transfer through flow energy cascading, hence enhancing the forced convection process. In general, the amalgamations of ( $\left.U_{\text {high, }} I_{\text {high }}, L_{\text {low }}\right)$ are determined to be the favourable characteristics for potent thermal transfer processes. Such numerical findings coincide closely with empirically detected flow dynamics at centreline $x / D_{h}=0.125$, justifying the current use of SPTV in describing for the positive thermal dissipation flow properties. Hence, statistical analysis is conducted subsequently for a rigorous analyzation on grid induced flow characteristics.

\section{Statistical analysis of turbulent flow}

With the empirical computation of tracer particle's velocity fluctuations, it is possible to calculate the acceleration of particle for each directional component with the formula below:

$$
a_{x}=\lim _{t_{2} \rightarrow t_{1}} \frac{u_{2}^{\prime}-u_{1}^{\prime}}{t_{2}-t_{1}} \quad a_{y}=\lim _{t_{2} \rightarrow t_{1}} \frac{v_{2}^{\prime}-v_{1}^{\prime}}{t_{2}-t_{1}} \quad a_{z}=\lim _{t_{2} \rightarrow t_{1}} \frac{w_{2}^{\prime}-w_{1}^{\prime}}{t_{2}-t_{1}}
$$

where, $a_{x}, a_{y}$, and $a_{z}$ represent the particle accelerations in the $(x, y, z)$ directions, respectively. Skewness $S$ and kurtosis $K$, which represent the symmetricity and the extremities of data distribution, are utilized to statically analyse the acceleration components induced from the 


$$
\begin{aligned}
& S=\frac{\left\langle a_{i}^{3}\right\rangle}{\left\langle a_{i}^{2}\right\rangle^{\frac{3}{2}}} \\
& K=\frac{\left\langle a_{i}^{4}\right\rangle}{\left\langle a_{i}^{2}\right\rangle^{2}}
\end{aligned}
$$

where, $i$ denotes the $(x, y, z)$ directional components.

Undeniably, $S=0$ and $K=3$ represent the Gaussian distribution of data. It is noticed in Fig. 8(a,b) that the acceleration distributions are rather normally distributed for NG and RG, but with a slight positive skew, where $S(\mathrm{NG})<S(\mathrm{RG})$ in all acceleration components. Such results signify an increase in accelerated flow for RG as compared to NG, especially in the streamwise direction. Clearly, a non-Gaussian behaviour is realized for SFG, PCRG and PCSFG, whereby $(|S|>0, K>3)$ are recorded for the former, and $(|S|>0, K<3)$ for the latter duo. The $\pm S$ achieved by SFG implies extreme decelerated turbulent events documented in the ( $x$, $y$ ) directions, along with turbulent accelerations in $z$-direction. These accelerations are considered to be rare and intensive, as indicated with the high positive $K>3$. Interestingly, similar $S$ developments are recorded for the partially-covered grids, but vastly disparate in $K$ as evident from Fig..8(b). The realization of $K \approx 2$ for PCRG and PCSFG depicts an increase in likelihood for the extreme decelerated $\left(a_{x}, a_{y}\right)$ turbulent events, which could very well imply the high occurrences of alternating flow directions that escalates to the formation of copious vortices.

Undoubtedly, PCSFG is seen to manifest the highest negative skewness of $S=-0.12$ for $a_{y}$, which may suggest the preference for exhibiting extreme decelerated flow structures in the lateral direction for a strong thermo-fluid interplay between highly potent and numerous collaborative vortices. The comparable negative $S\left(a_{x}, a_{y}\right)$ indicated the small $L$ eddies are compacted with intense $X-Y$ plane flow circulations. Such high occurrences of dense and powerful eddies accompanied with minor vertical accelerations enable an extended exposure to the reshuffling of fins' boundary layer, thus generating highly positive thermal dissipation flow structures. Even though PCRG exhibits greater negative $S\left(a_{x}\right)$, it is deprived in the vital negative $S\left(a_{y}\right)$, consequently dampens the strength of flow circulation and forced convection capabilities. Nevertheless, the greater displays of such events as compared with SFG allow for $\mathrm{Nu}(\mathrm{PCRG})>\mathrm{Nu}(\mathrm{SFG})$, even though the numeral polarity of $S$ registered similarity. Conversely, $\mathrm{NG}$ and RG demonstrated moderate flow circulations, hence the low $\mathrm{Nu}$. In general, the extreme decelerated flow events are capable of forming intense flow vortices, which is 
beneficial in disruption of fins' boundary layer. Further research is still required to uncover the $S$ and $K$ profiles at different inter-fin locality, in order to uncover the overall flow structures that are preferable for maximising forced convection of plate-fin heat sink.

Power spectra density (PSD)

When comparing the effects of various grids in their heat transfer performance, it is relevant to consider the PSD of flow, which deciphers the strength of velocity fluctuations in accordance with the frequency domain. Such feat is achieved by utilizing Fourier transform on the non-normalized autocovariance function of velocity fluctuation with respect to time, and is defined as follow:

$$
\begin{gathered}
P(f)=\int_{-\infty}^{\infty} r_{f}(t) e^{-j 2 \pi f t} d t \\
r_{f}(t)=\left\langle f^{\prime}(t) f^{\prime}(t+\tau)\right\rangle
\end{gathered}
$$

where $P(f)$ represents the PSD as a function of frequency $f, r_{f}$ the non-normalized autocovariance function of velocity fluctuations and $j$ the imaginary unit. The PSDs achieved by the $2 \mathrm{D}$ planar grids under influence of $\mathrm{Re}_{D h}=22 \times 10^{3}$ are illustrated in Fig. 9. It is worthwhile to mention that the profile was subjected with 10-lapse period of moving average to minimize random noises.

Clearly, the energy level demonstrated by the partially-covered grids are much higher compared to the fully-covered counterparts, i.e. an impressive increase of approximately $\times 10^{2}$, with NG recorded at the lowest energy state. It is noticed that the $P(f)$ of all grid configurations decreases with increasing $f$, but proceed to surge at higher frequency domains, thereupon a second stage decay. Interestingly, the initial $P(f)$ decay regions exhibit a reduction profile similar to that of Kolmogorov-law, i.e. with exponent approaching $-5 / 3$. Most grids are observed to follow the decay exponent over a wide range of frequencies except for PCSFG, where the $f^{-5 / 3}$ is only documented at a narrow band, i.e. approximately between $4 \mathrm{~Hz}<f<7 \mathrm{~Hz}$. Such dissimilarity recorded for PCSFG might indicate the formation of a distinct and unique flow structure that could potentially maintain an intense energy level over wider range of frequencies, which could be beneficial for superior thermal transfer.

As previously mentioned, the energy level surges at higher frequency ranges, and is exceptionally pronounced for the partially-covered planar grids. The vertically aligned mid- 
513 plane separation gives rise to the formation of dynamic coherent flow structures, thus inducing 514 a distinct velocity fluctuation. Such wake-flow interactions result in a powerful vortex shedding 515 effect, which justifies the excitation at $P(f)$ peak. Moreover, the raised energy levels are 516 observed to span over a wide range of frequencies, with range of PCSFG>PCRG. The 517 phenomenon may imply that partially-covered grids are capable of generating broad array of 518 high energy multilength scale eddies through (i) first stage grid-separation induced turbulence and (ii) second stage plate-fin eddies filtration for an intense vortex shedding process. As PCSFG comprised of different fractal bar thicknesses, there would be greater variations in eddies length scale, hence ampler variety of frequencies. Contrariwise, utilization of fullycovered grids masked the powerful vortex shedding effect, and is further subdued under NG configuration. Surprisingly, the energy profile demonstrated by NG and RG are very much identical, revealing the impact of 2D planar grids in raising the preferable flow energy levels for forced convective heat transfer.

Upon closer inspections, it is noticed that the $P(f)$ peaks are recorded at different frequencies, with the lowest realization of $f=18.45 \mathrm{~Hz}$ by PCSFG, thereupon PCRG, SFG, NG, and RG ranked in ascending order. The differences in frequencies might suggest the presence of an optimum fluctuation frequency $f_{\lambda}$ that maximizes heat transfer through forced convection in plate-fin heat sink. As suggested $\mathrm{in}^{17}$, that velocity fluctuation frequencies at the two extremes of $f_{\lambda}$ are ineffective in thermal transfer, as high frequencies $\left(f \mid f_{\lambda} \gg 1\right)$ contributed to diffusive effects, whereas low frequencies $\left(f / f_{\lambda} \ll 1\right)$ appeared as quasi-steady. Moreover, flow fluctuations at high frequency sub-range may be too rapid for any reshuffling of boundary layers to take effect. The particle's trajectory projection illustrated in Fig. 6(a, b) supports the preceding statements, as high frequency fluctuations by PCRG and SFG appear to develop diffusive characteristics in the time-lapsed particle trajectory, causing the extensive contribution of turbulent kinetic energy confined around the shedding frequency to be ineffective in enhancing plate-fin heat transfer. As for PCSFG, the $f$ is presumed to be approaching $f_{\lambda}$, thus resulting in the formation of dense and intense annulus trajectory that directs the turbulent kinetic energy along the boundary layer for maximum agitation. In short, $f_{i} \approx 18.45 \mathrm{~Hz}$ provides adequate velocity fluctuations for direct boundary layer restructuring, whist containing sufficient flow momentum to effectively enhance thermal dissipation.

\section{Conclusion}

An in-house developed SPTV system was utilized to investigate the underlining gridinduced flow structures that reinforce positive thermal dissipation of plate-fin array, which was 
546 numerically optimized at $\delta=5 \mathrm{~mm}$ and $l=10 \mathrm{~mm}$ under $\operatorname{Re}_{D h}=22.0 \times 10^{3}$. The $2 \mathrm{D} \mathrm{Nu}$ contour plots

547 were able to reveal augmentations to heat transfer using partially-covered grids, where high $\mathrm{Nu}$

548 was seen at wider coverage range of $(\delta, l)$. Furthermore, the increases of $42.9 \%$ and $29.2 \%$ in

$549 \mathrm{Nu}$ were observed by PCSFG and PCRG, respectively, as compared to control NG. Such

550 enhancements were believed to be stimulated from preferable flow of (i) high $U / U_{0}$, (ii) intense

$551 I$, (iii) strong $\left(v^{\prime}, w^{\prime}\right)$, (iv) small $L / \delta$, and (v) negative $S\left(a_{x}, a_{y}\right)$ with (vi) low $K$ for maximum

552 thermal dissipation. These coupling flow dynamics also developed a unique formation of

553 'Turbulent Annulus' which offers a small degree of predictability on turbulence in the periodic

554 annulus oscillation that effectively reshuffle flow boundary layers. Lastly, the PSDs induced

555 by partially-covered grids were of higher orders, with a prominent peak at high frequency sub-

556 range that indicates strong vortex shedding effect. An optimal frequency of $f_{\lambda} \approx 18.45 \mathrm{~Hz}$ was

557 determined by PCSFG, which was believed to provide adequate turbulent kinetic energy for

558 direct boundary layer restructuring, while maintaining the necessary flow momentum to ensure

559 superior force convective heat transfer of plate-fin heat sink. 
561 1. Bailly, C. \& Comte-Bellot, G. Turbulence 1-5 (Cham: Springer International Publishing, 562 2015).

563 2. Hurst, D. \& Vassilicos, J.C. Scalings and decay of fractal-generated turbulence. Phys. Fluids 19, 035103 (2007).

565 3. Cafiero, G. Greco, C.S. Astarita, T. \& Discetti, S. Flow field features of fractal impinging jets at short nozzle to plate distances. Exp. Therm. Fluid Sci. 78, 334-344 (2016).

4. Cafiero, G. Castrillo, G. Greco, C. S. \& Astarita, T. Effect of the grid geometry on the convective heat transfer of impinging jets. Int. J. Heat Mass Transf. 104, 39-50 (2017).

5. Cafiero, G. Castrillo, G. \& Astarita, T. Turbulence properties in jets with fractal grid turbulence. J. Fluid Mech. 915, A12 (2021).

6. Verbeek, A.A. Willems, P.A. Stoffels, G.G.M. Geurts, B.J. \& van der Meer, T.H. Enhancement of turbulent flame speed of V-shaped flames in fractal-grid-generated turbulence. Combust. Flame 167, 97-112 (2016).

7. Ferko, K. Chiappazzi, N. Gong, J. \& Danesh-Yazdi, A.H. Power output comparison of side-by-side fluidic harvesters in different types of fractal grid-generated turbulence. In: Active and Passive Smart Structures and Integrated Systems XIII (ed Erturk A). Spie-Int Soc Optical Engineering (2019).

8. Ferko, K. Chiappazzi, N. Gong, J. \& Danesh-Yazdi, A.H. Average power output and the power law: identifying trends in the behavior of fluidic harvesters in grid turbulence. In: Active and Passive Smart Structures and Integrated Systems XIII (ed Erturk A). Spie-Int Soc Optical Engineering (2019).

9. Melina, G. Bruce, P.J.K. Hewitt, G.F. \& Vassilicos, J.C. Heat transfer in production and decay regions of grid-generated turbulence. Int. J. Heat Mass Transf. 109, 537-554 (2017).

10. Mazellier, N. \& Vassilicos, J.C. Turbulence without Richardson-Kolmogorov cascade. Phys. Fluids. 22, 1-25 (2010).

11. Seoud, E.R. \& Vassilicos C.J. Dissipation and decay of fractal-generated turbulence. Phys. Fluids 19, 105108 (2007).

12. Teh, A.L. Phoo, Y.W. Chin, W.M. Ooi, E.H. \& Foo J.J., Forced convective heat transfer enhancement of $90^{\circ}$ bend plate-fin heat sink with grid generated turbulence. Chem. Eng. Res. Des. 156, 226-239 (2020). 
13. Hoi, S.M. Chew, I.M.L. Ooi, E.H. \& Foo, J.J. SPTV sheds light on fluid dynamics of fractal-induced turbulence over a plate-fin array forced convection. Exp. Therm. Fluid Sci. under Rev. (2020).

14. Hoi, S.M. Teh, A.L. Ooi, E.H. Chew, I.M.L. \& Foo, J.J. Plate-fin heat sink forced convective heat transfer augmentation with a fractal insert. Int. J. Therm. Sci. 142, 392406 (2019).

15. Hoi, S.M. Teh, A.L. Ooi, E.H. Chew, I.M.L. \& Foo, J.J. Forced convective heat transfer optimization of plate-fin heat sink with insert-induced turbulence. Appl. Therm. Eng. 160, 114066 (2019).

16. Omilion, A. Turk, J. \& Zhang, W. Turbulence enhancement by fractal square grids: effects of multiple fractal scales. Fluids 3, 37 (2018).

17. Dullenkopf, K. \& Mayle, R.E. An account of free-stream-turbulence length scale on laminar heat transfer. J. Turbomach. 117, 401-406 (1995).

18. Torii, S. \& Fuse, H. Effects of the length scale of free-stream turbulence and cylinder size on heat transfer in laminar separated flows. Exp. Therm. Fluid Sci. 619-624 (1993).

19. Sak, C. Liu, R. Ting, D.S.K. \& Rankin, G.W. The role of turbulence length scale and turbulence intensity on forced convection from a heated horizontal circular cylinder, Exp. Therm. Fluid Sci. 31, 279-289 (2007).

20. ANSYS® Academic Research, ANSYS fluent theory guide. Release 15.0 (2014). 


\section{Acknowledgement}

612 The authors would like to express their gratitude to the Malaysian Ministry of Higher Education

613 (MOHE) for the financial support of the present research investigation

614 (Project Code: FRGS/1/2018/TK07/MUSM/02/1). The author would also like to gratefully

615 acknowledge Monash University Malaysia (MUM) for the financial support of the current

616 research project (MUM25929267).

\section{Author Contributions}

618 S.H.C. prepared the initial draft, S.M.H., M.V.T. and J.J.F. revised, refined and edited the 619 manuscript. All authors reviewed the manuscript.

620 Competing Interest

621 The authors declare no competing interests.

622 Additional Information

623 Correspondence and requests for materials should be addressed to S.H.C. or J.J.F. 


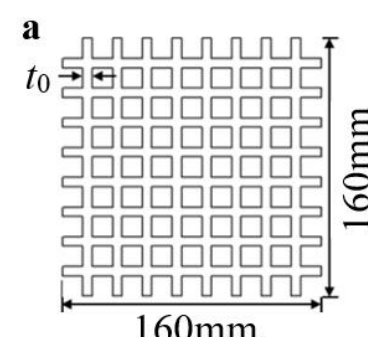

$160 \mathrm{~mm}$

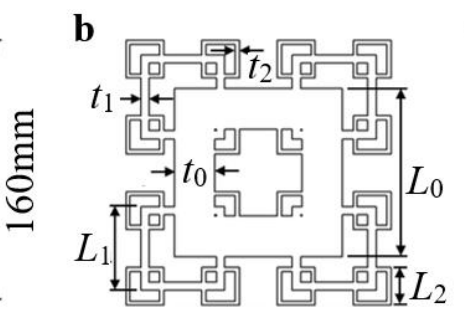

b c

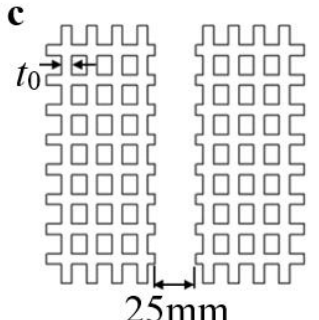

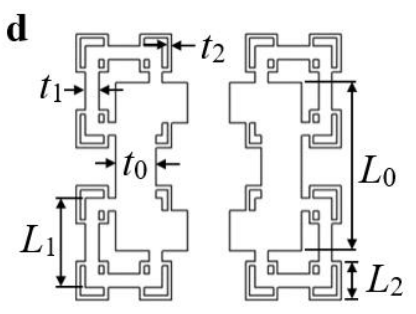

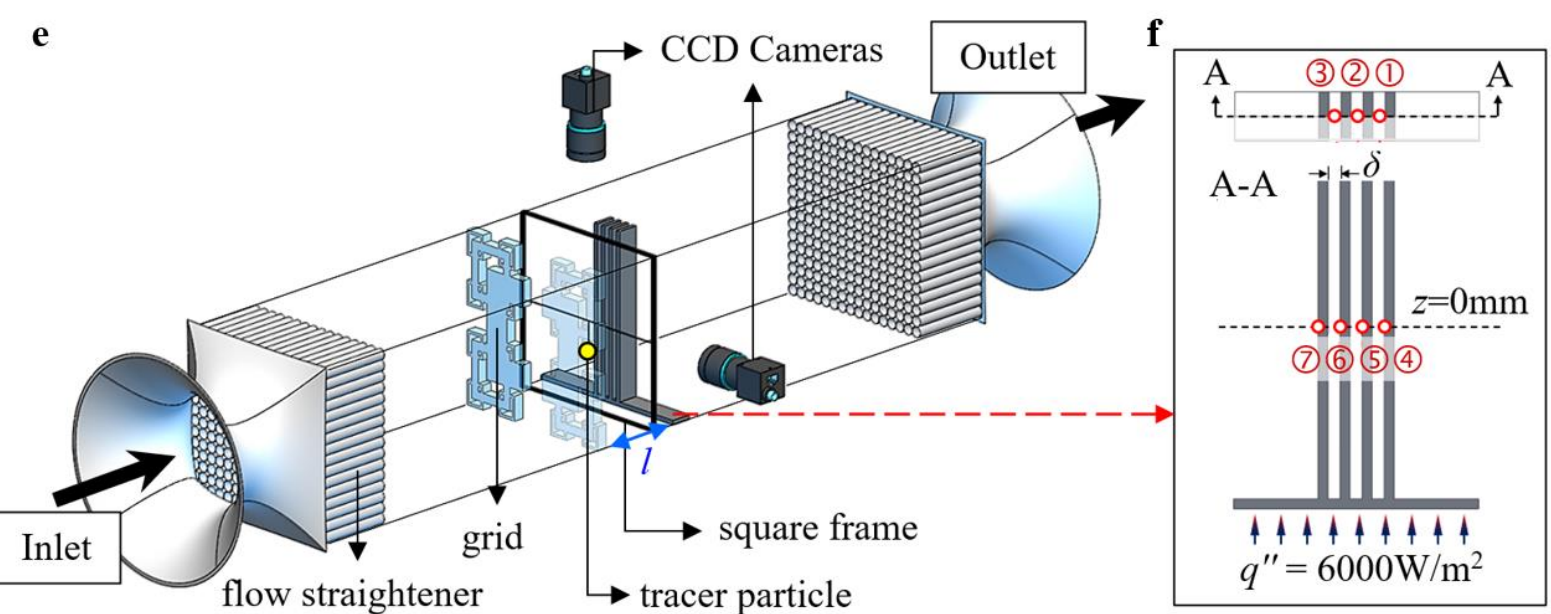

Fig. 1: Schematic diagram of 2D planar space-filling gird of (a) RG, (b) SFG, (c) PCRG, (d) PCSFG, (e) geometrical representation of wind tunnel test section with tracer particle attached on square frame and (f) seven $T$-type thermocouple locations on the plate-fin heat sink. 

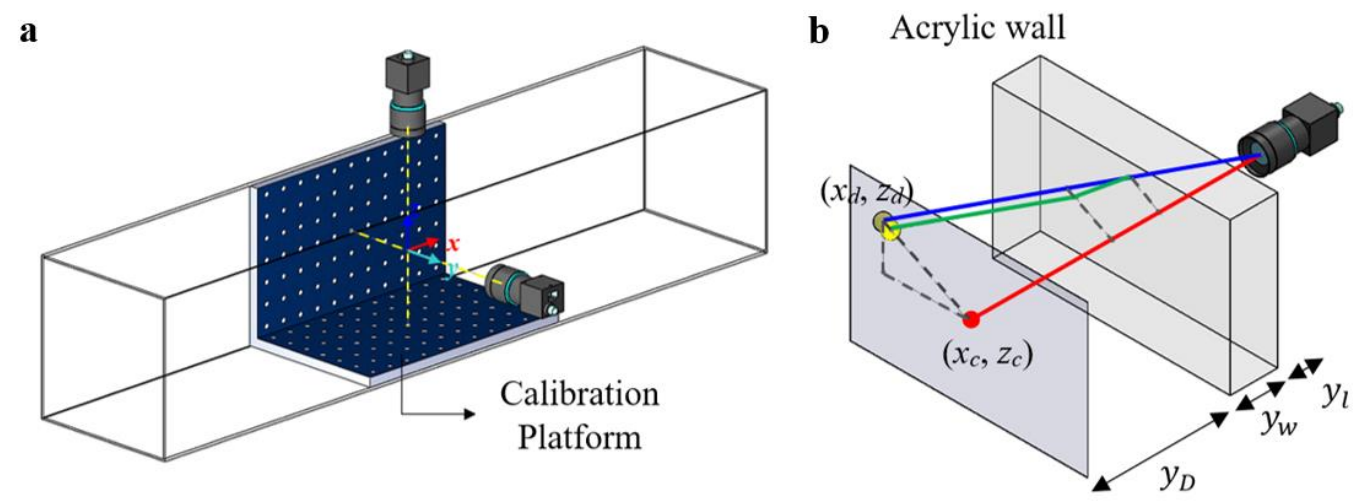

c

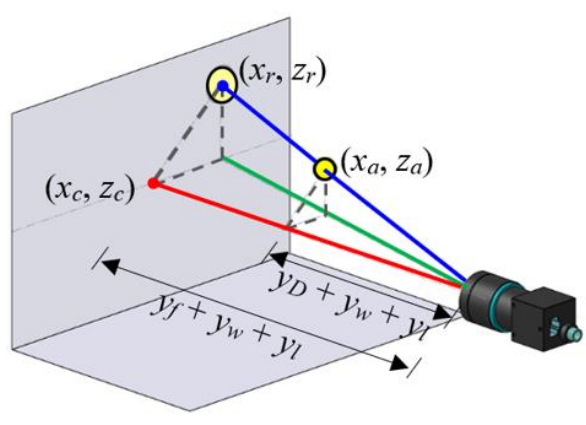

d

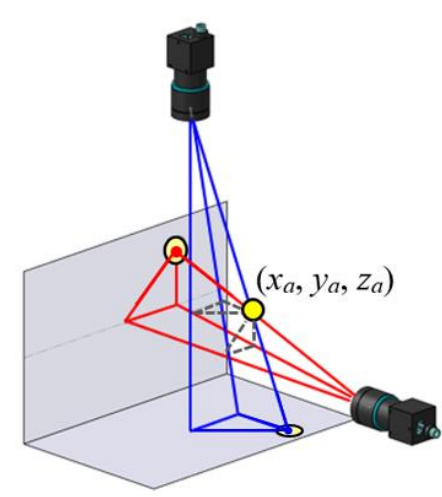

$\mathbf{e}$

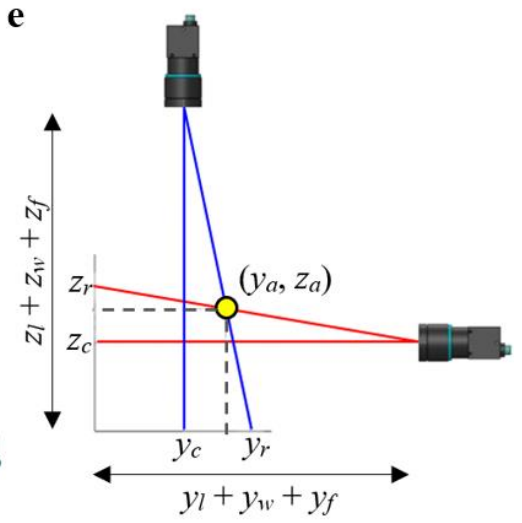

Fig. 2: (a) Alignment of cameras using $7 \times 11$ circle array calibration platform, with the Cartesian coordinate set at intersection of cameras' optical axis. (b, c) demonstrate the notation used for refractive and perspective error correction processes. Note that the perspective error influences the (d) projected coordinates of the two cameras, and (e) is the corresponding Y-Z view of the projection. 


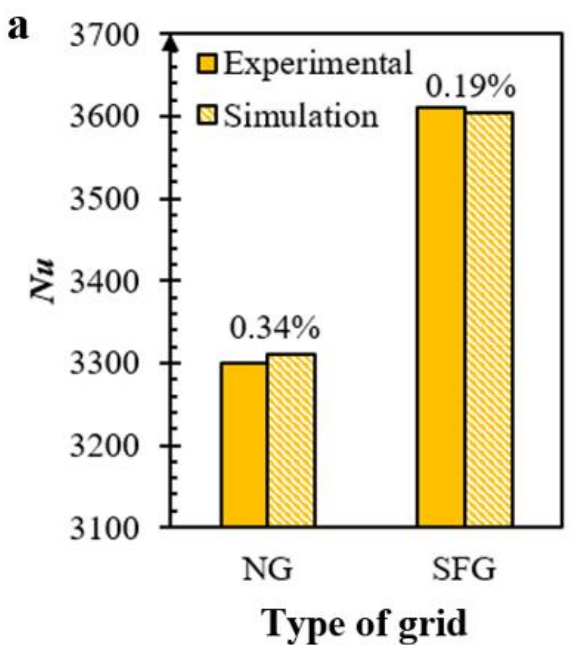

c

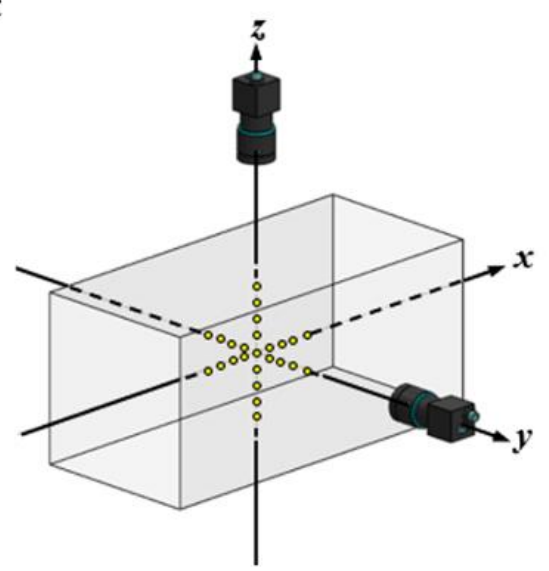

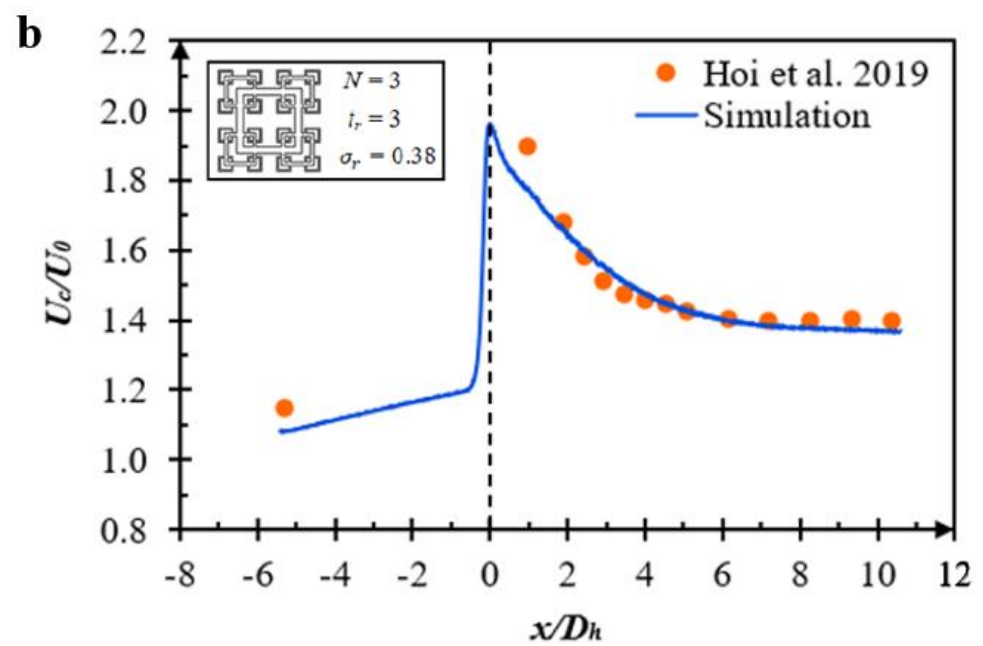

d

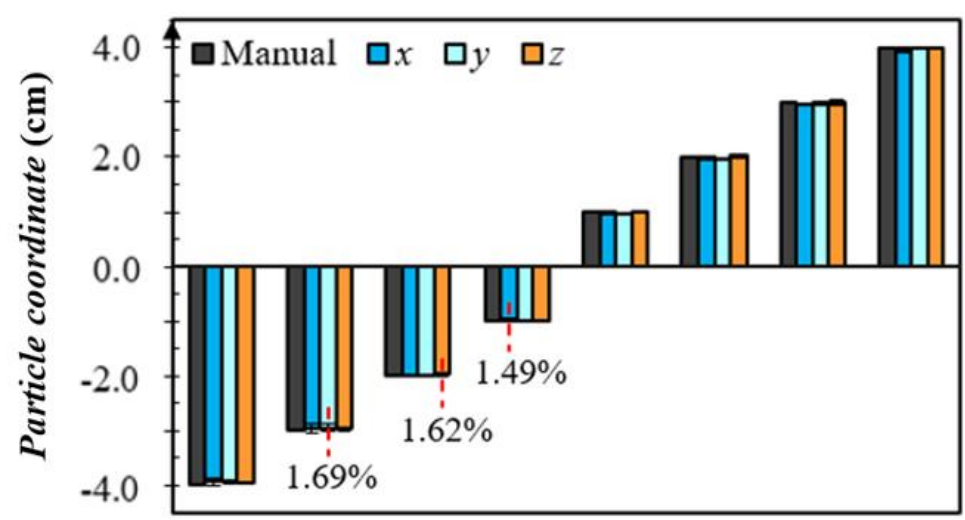

Fig. 3: Top, numerical validation of (a) $N u$ for NG and SFG, as well as (b) the normalized centreline velocity of fractal grid generated turbulence against Hoi et al. experimental data, viz. [13] and [14], respectively. Bottom, (c) geometrical representation of particle placements in the wind tunnel test section along with (d) the SPTV recorded error margins. 

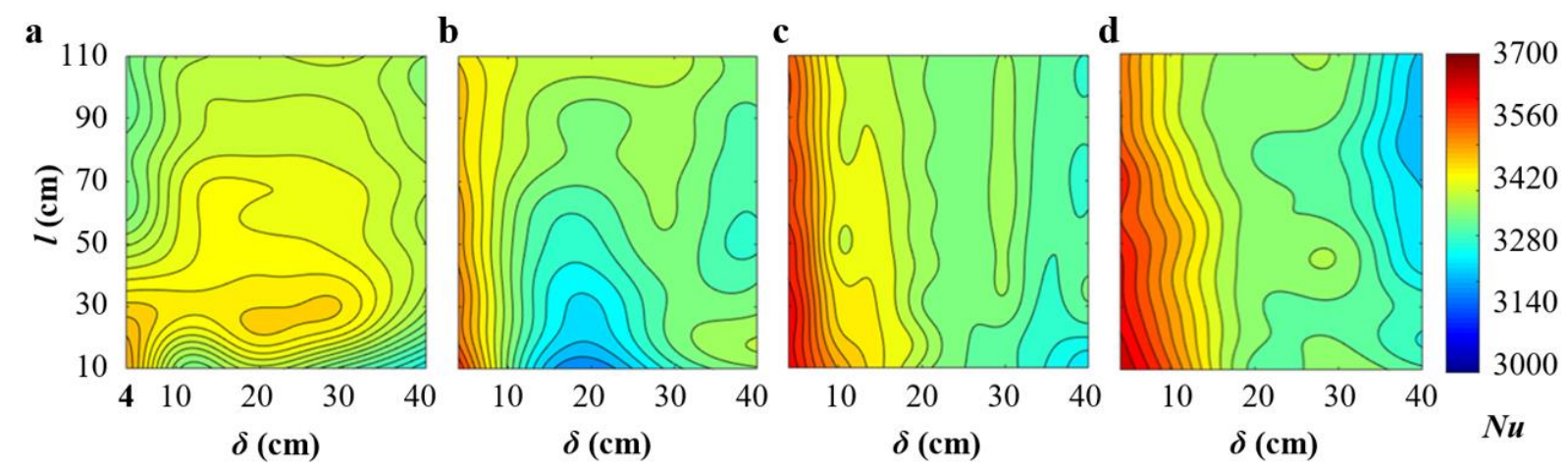

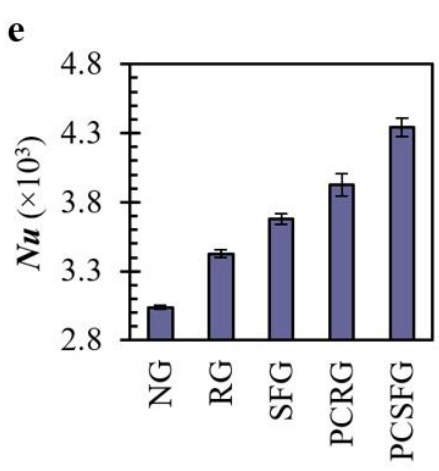

Type of grid

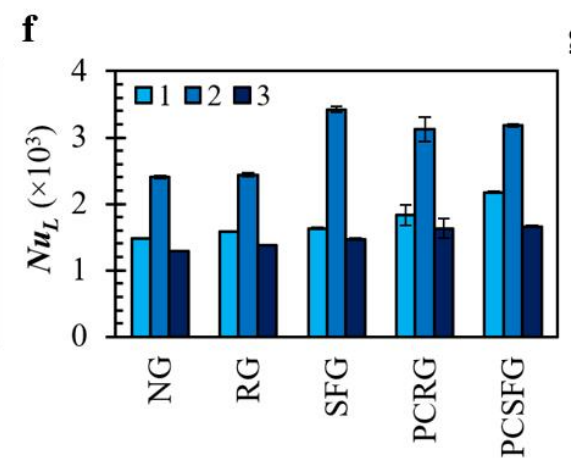

Type of grid

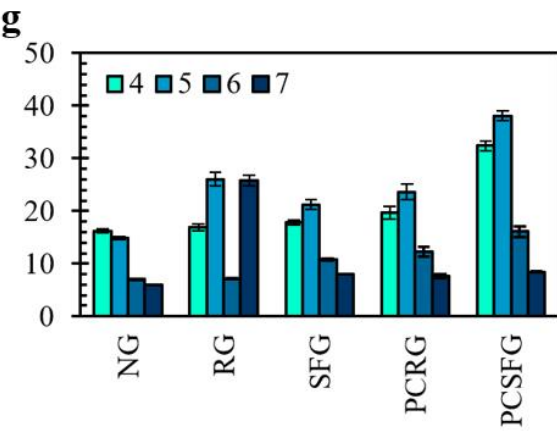

Type of grid

Fig. 4: Top, the numerical 2D Nu contour plot induced by (a) RG, (b) PCRG, (c) SFG and (d) PCSFG as a function of $l$ against $\delta$. Bottom, various grid empirically induced (e) $N u$, along with the $N u_{L}$ of (f) base section and (g) mid-fin section of plate-fin heat sink. 


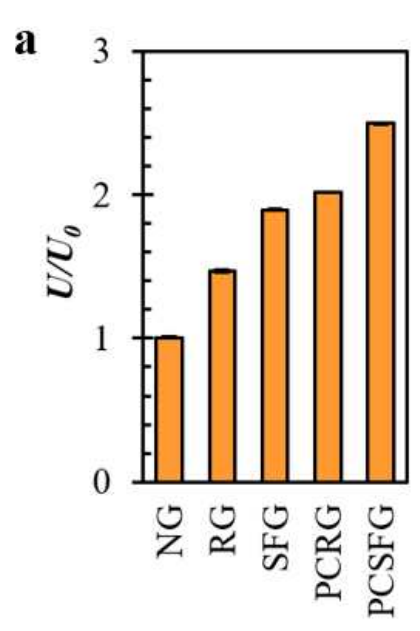

Type of grid

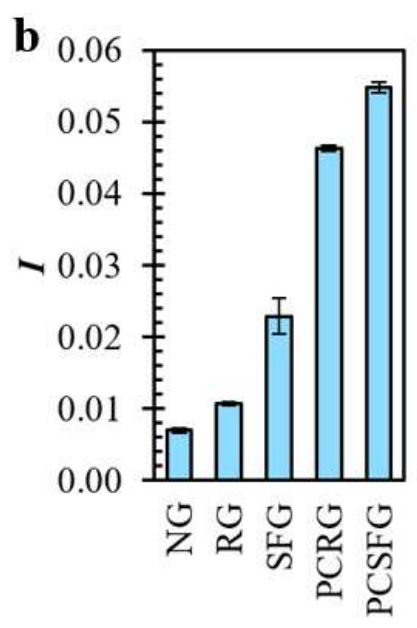

Type of grid

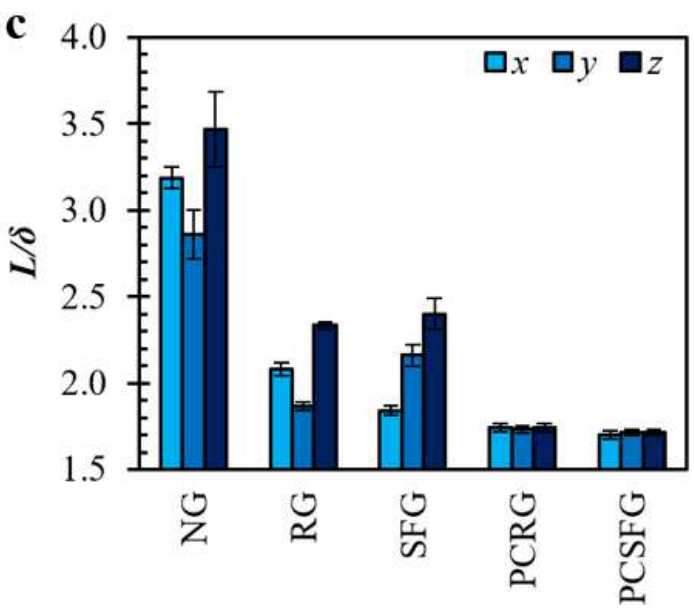

Type of grid

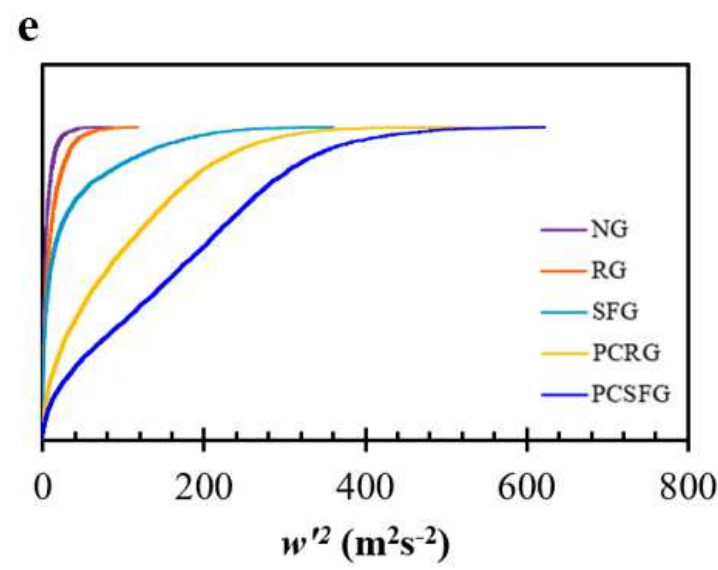

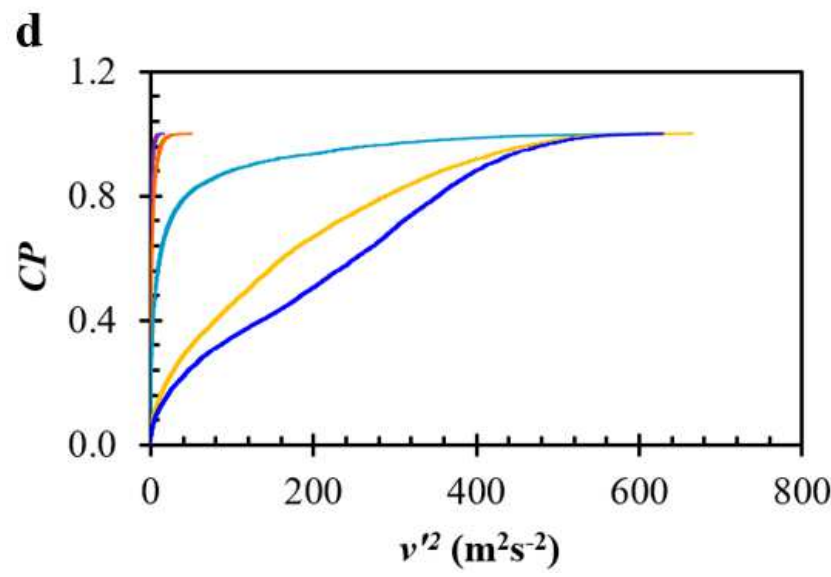

Fig. 5: Various grid induced (a) $U / U_{0}$, (b) $I$, (c) normalized $L / \delta$ in $(x, y, z)$ directional components at centreline $x / D_{h}=0.125$ and cumulative probability distributions of (d) $v^{\prime 2}$ and (e) $w^{2}$. 

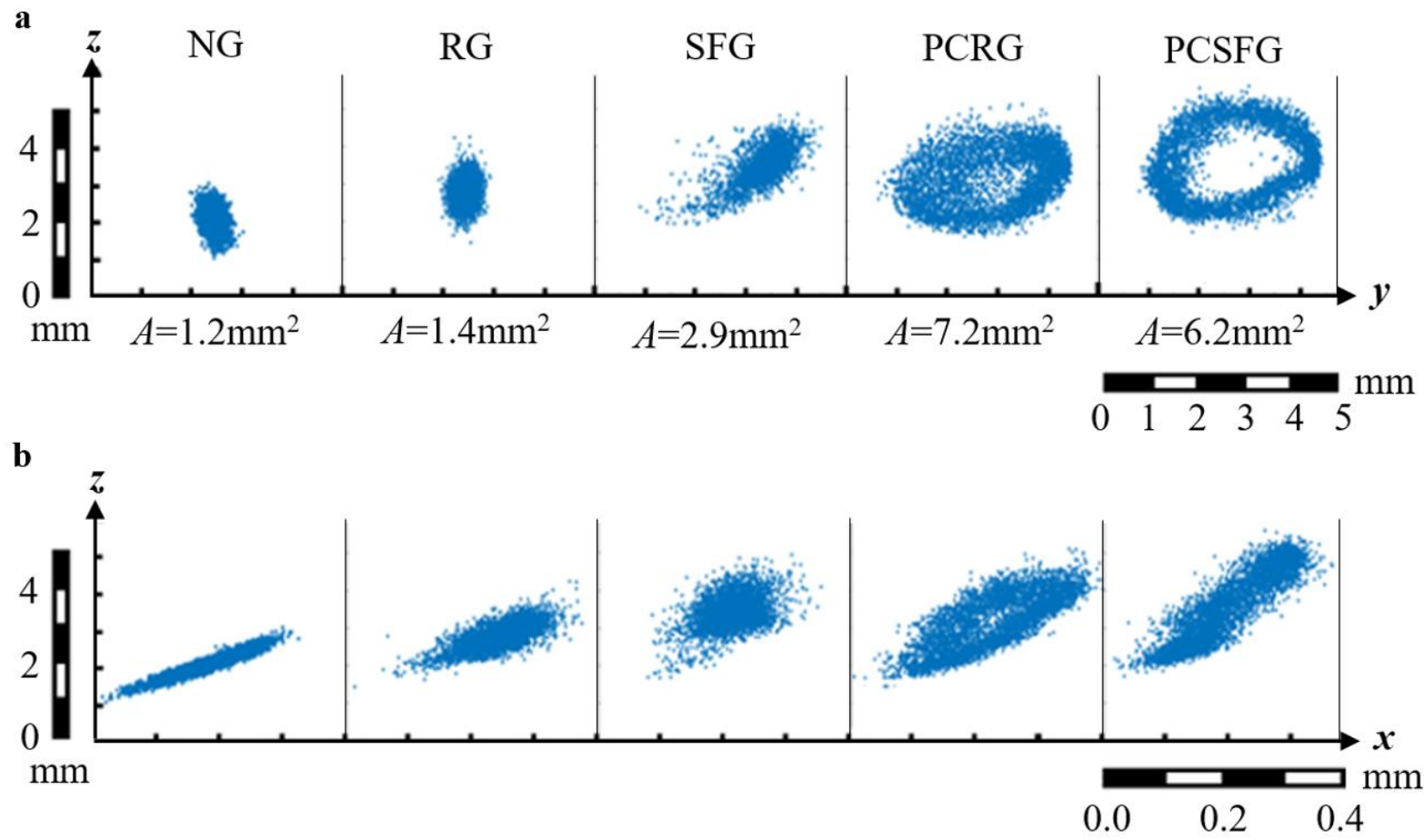

Fig. 6: Trajectory of SPTV tracer particle in the (a) cross-sectional and (b) side view induced from various grid turbulence. 

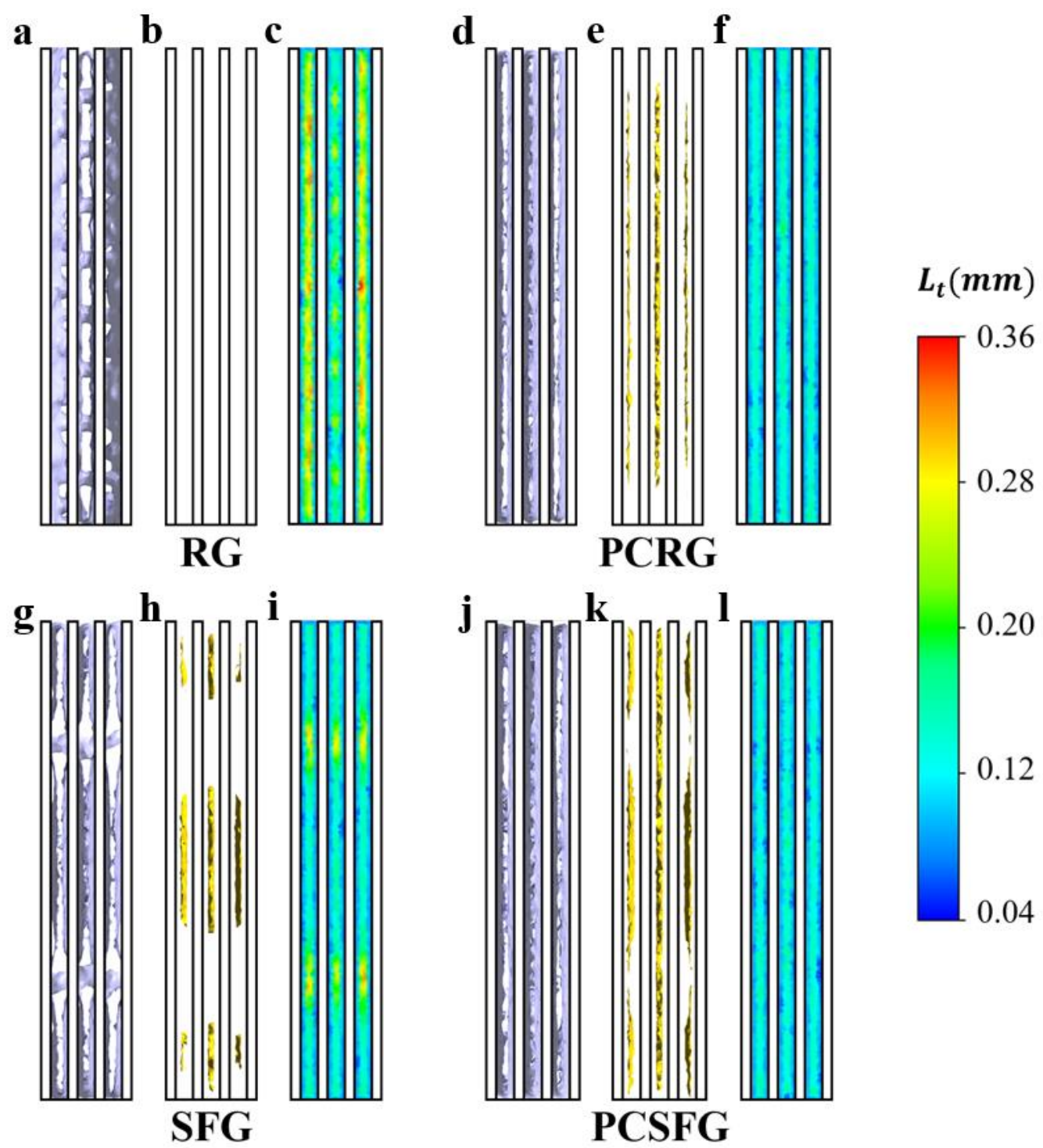

Fig. 7: The cross-sectional isosurface representation of (a, d, g, j) $U / U_{0}=2.38$, (b, e, h, k) $I=0.33$; and $(\mathrm{c}, \mathrm{f}, \mathrm{i}, \mathrm{l})$ the $2 \mathrm{D}$ contour plot of $L$ induced from various grid at $x / \mathrm{Dh}=0.125$. Note that $\delta=5 \mathrm{~mm}$ and $l / D_{h}=0.0625$. 
a

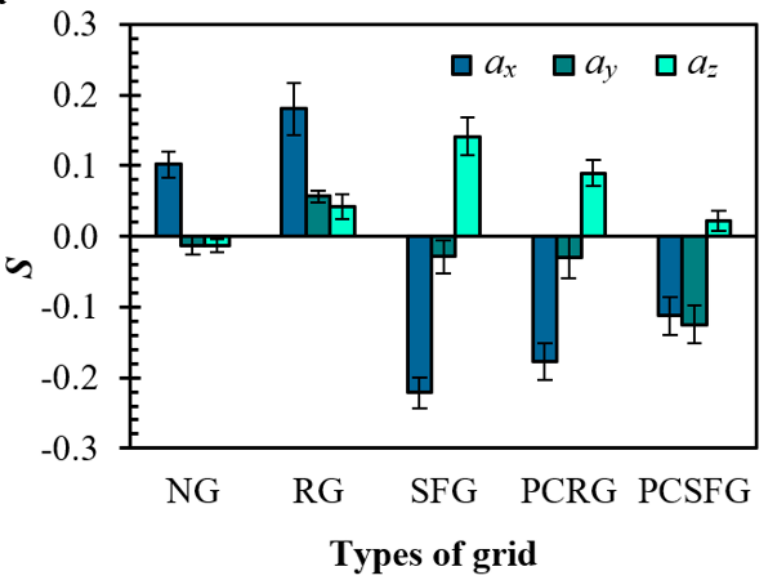

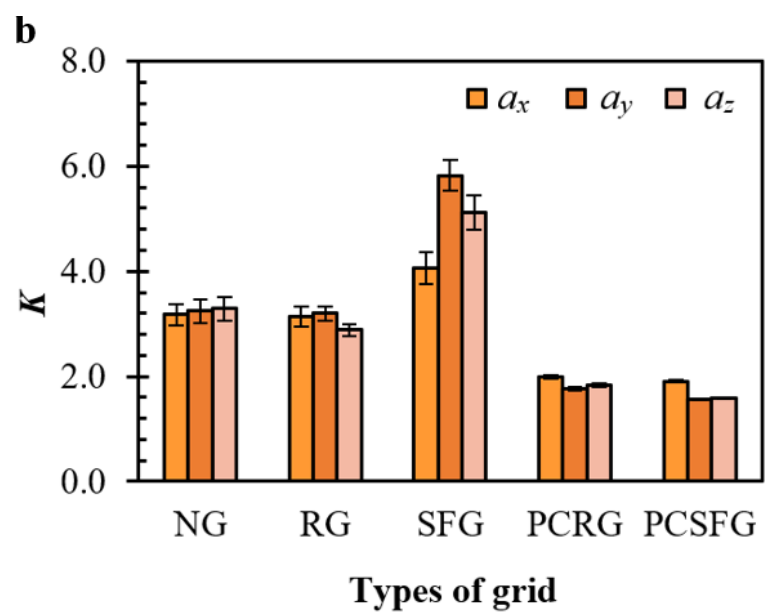

Fig. 8: Various grid induced turbulence on (a) $S$ and (b) $K$ of SPTV particle acceleration in the $(x, y, z)$ directional components. 


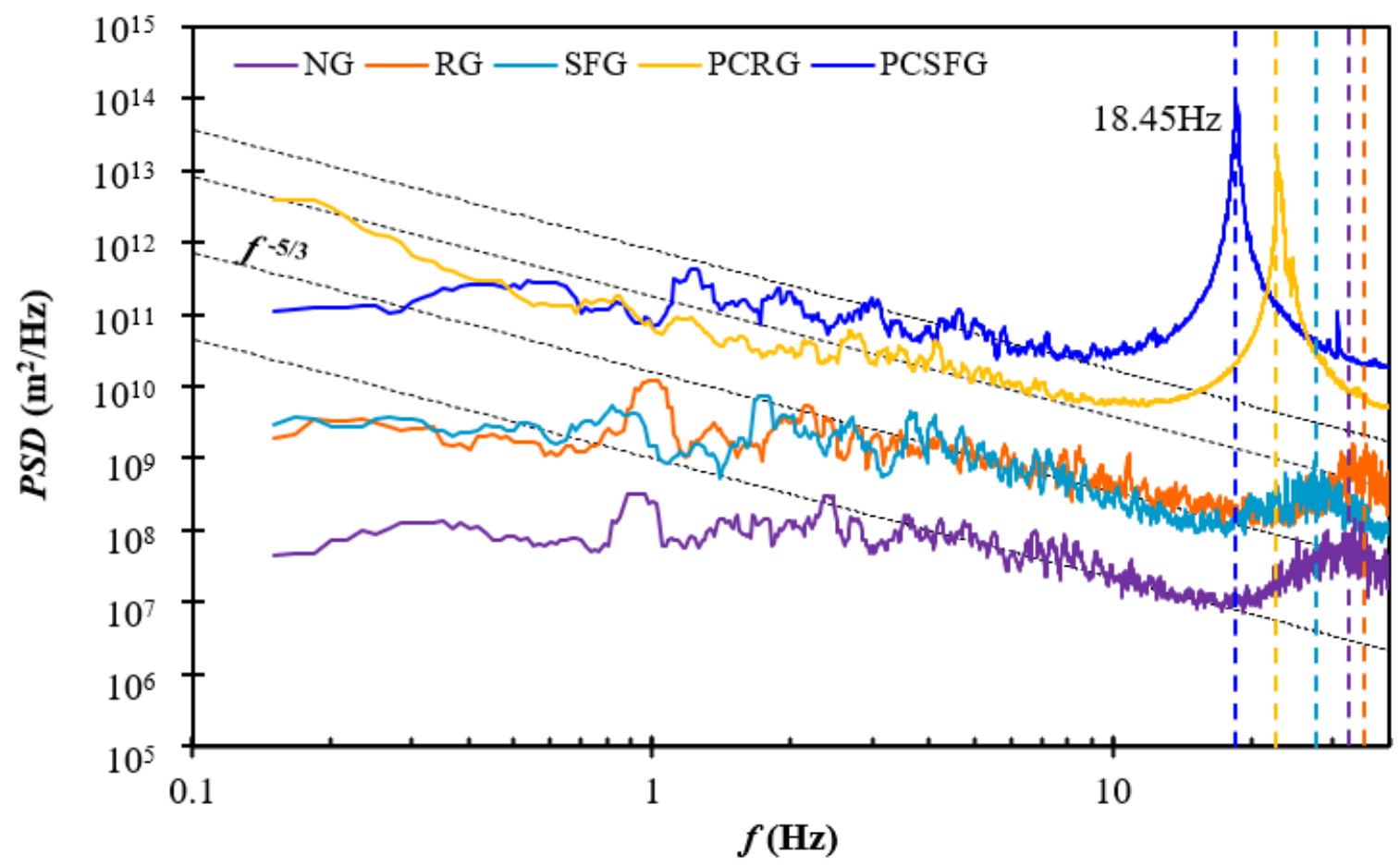

Fig. 9: The 10-lapse period moving averaged PSD of velocity fluctuation induced with various grids at $R e_{D h}=22.0 \times 10^{3}$. 